\title{
DENSITY PERTURBATIONS IN AN UNIVERSE DOMINATED BY THE CHAPLYGIN GAS
}

\author{
J. C. Fabris', S.V.B. Gonçalves[ and P.E. de Souza \\ Departamento de Física, Universidade Federal do Espírito Santo, CEP29060-900, \\ Vitória, Espírito Santo, Brazil
}

\begin{abstract}
We study the fate of density perturbations in an Universe dominate by the Chaplygin gas, which exhibit negative pressure. We show that it is possible to obtain the value for the density contrast observed in large scale structure of the Universe by fixing a free parameter in the equation of state of this gas. The negative character of pressure must be significant only very recently.

PACS number(s): 98.80.Bp, 98.65.Dx
\end{abstract}

The nature of "missing mass" that must dominate the Universe today is one of the most intriguing problem in modern physics. This problem appears at various levels, beginning with the rotation curve of spiral galaxies [1], and extending to the total mass of the Universe as it is deduced through the first acoustic peak of the spectrum of the anisotropy of cosmic background radiation [2]. A fraction of this missing mass has been called dark energy since it does cluster, remaining a smooth component in the Universe. This problem has acquired a most dramatic face through the results coming from the observation of supernova type Ia, which indicate that the Universe today is in an accelerated expansion [3, 4. Hence, the inflationary paradigma, first conceived for the primordial Universe, can be applicable to our actual Universe.

In this way, the cosmological constant problem was revived: it is possible that a cosmological constant may be the dominant component of matter in the Universe. However, other types of matter with negative pressure have been considered as candidate of the so-called dark energy of the Universe: fluids of topological defects (domain walls, strings), quintessence (a self-interacting scalar field minimally coupled to gravity), etc. Recently, a different matter component with negative pressure has been presented as possible candidate for this dark energy: the Chaplygin gas [5].

The Chaplygin gas has an equation of state of the type

$$
p=-\frac{A}{\rho},
$$

where $A$ is a constant. This exotic fluid has an interesting origin. Taking the NambuGoto action of string theory, the Chaplygin fluid appears after considering $d$-branes in a

\footnotetext{
${ }^{1}$ e-mail: fabris@cce.ufes.br

${ }^{2}$ e-mail: sergio@cce.ufes.br

${ }^{3}$ e-mail: patricia.ilus@bol.com.br
} 
$d+2$ dimensional space-time, in the light cone gauge [6]. In this case, the Nambu action reduces to [7]

$$
S=\int d^{d+1} r\left[\theta \dot{\rho}-\left(\rho(\nabla \theta)^{2}+\frac{1}{2} \frac{A}{\rho}\right)\right] .
$$

A very curious aspect of this action is that the variables $\theta$ and $\rho$ obey the newtonian hydrodynamic equations for an irrotational fluid with the pressure given by (1). Moreover, the Chaplygin gas admit a supersymmetric extension.

In [5], the consequences for the evolution of the Universe of such exotic fluid have been investigate. They found that, in the relativistic context, the density is connected to the scale factor as

$$
\rho=\sqrt{A+\frac{B}{a^{6}}} .
$$

Hence, initially the Universe behaves as it was dominated by a dust fluid, and than the density becomes asymptotically constant, revealing that the cosmological constant becomes the dominant component of the Universe. There is an intermediate phase, which can be described by a cosmological constant mixed with a stiff matter fluid. In this sense, this exotic fluid may be a candidate for describing the Universe as the supernova observational results indicate it must be.

In the presente note, we study another aspect of this fluid: the evolution of density perturbations. A relativistic study of this problem leads to some technical difficulties. Considering that we can approximate the Universe filled by such a fluid with the three phases described before, the behaviour of density perturbation in the initial and final phases are already known $\left(\delta_{\text {dust }} \propto t^{2 / 3}\right.$ and $\delta_{c c}=0$ respectively). Hence in order to obtain non-trivial effects we must consider the intermediate phase. However, such a phase admits solution for the background, but no analytical solution for the perturbed equations.

Since our intention is to have explicity expression for density perturbations, in order at least to evaluate what happens for the evolution of structure in an Universe dominate by the Chaplygin gas, we will exploit the fact that the action (2) leads to the hydrodynamic newtonian equations. Coupling to (2) a term representing the gravitational potential $\phi$ we have the following equations [8]:

$$
\begin{aligned}
\frac{\partial \rho}{\partial t}+\nabla \cdot(\rho \vec{v}) & =0, \\
\dot{\vec{v}}+\vec{v} \cdot \nabla \vec{v} & =-\frac{\nabla p}{\rho}+\nabla \phi, \\
\nabla^{2} \phi & =-4 \pi G \rho .
\end{aligned}
$$

If these equations describe an expanding homogeneous and isotropic Universe their solution is

$$
\rho=\frac{\rho_{0}}{a^{3}} \quad, \quad \vec{v}=\vec{r} \frac{\dot{a}}{a} \quad, \quad \nabla \phi=-\vec{r} \frac{4 \pi G \rho}{3} .
$$

Notice that these solutions are independent of the pressure. Indeed, since in a homogeneous Universe $p=p(t)$, there is no pressure gradient, and the presence of pressure does not influence the evolution of the Universe. 
We turn now to the perturbative level. Introducing in equations $(4,10,6)$ the quantities $\rho=\rho_{0}+\delta \rho, \vec{v}=\vec{v}_{0}+\delta \vec{v}, p=p_{0}+\delta p$ and $\phi=\phi_{0}+\delta \phi$, where each quantity has been expressed as a sum of the background solution plus a small perturbation, we can combine the resulting perturbed equation in order to obtain an unique differential equation for the density contrast $\frac{\delta \rho}{\rho}$ :

$$
\ddot{\delta}+2 \frac{a}{a} \dot{\delta}+\left\{\frac{v_{s}^{2} n^{2}}{a^{2}}-3 \frac{\ddot{a}}{a}\right\} \delta=0
$$

In this expression $v_{s}^{2}=\frac{\partial p}{\partial \rho}$ is the the sound velocity and $n$ is the wavenumber of the perturbation, which appears due to the fact that we expanded the spatial dependence of the perturbed quantities in plane waves. An important property of the Chaplygin gas is that, in spite of exhibiting negative pressure, its sound velocity is positive. This does not happen in general: fluids with negative pressure quite frequently exhibit instabilities, at least in their hydrodynamical representation, due to an imaginary sound velocity [9, 10].

Inserting the background solutions and the expression for the sound velocity in (8), we determine the behaviour of density perturbations. The solution reads

$$
\delta=t^{-1 / 6}\left[C_{1} J_{\nu}\left(\Sigma^{2} n t^{7 / 3}\right)+C_{2} J_{-\nu}\left(\Sigma^{2} n t^{7 / 3}\right)\right]
$$

where $\nu=5 / 14$ and $\Sigma^{2}=\frac{54}{49} \frac{A \pi G}{a_{0}^{2}}$. In principle $C_{1}$ and $C_{2}$ are constants that depend on $n$.

The solution (9) has two asymptotic regime. For $t \rightarrow 0$, there is a growing mode given by $\delta_{+} \propto t^{2 / 3}$. This coincides with the evolution of density perturbations in a dust fluid in General Relativity. On the other hand, for $t \rightarrow \infty$, the density contrast oscillates with decreasing amplitude, $\delta_{ \pm} \propto t^{-4 / 3} \cos \left(\Sigma^{2} n t^{7 / 3}+\phi\right)$, where $\phi$ is a phase, approaching a zero value asymptotically. Hence, for large values of time, the solution approaches the result for density perturbation in General Relativity with a cosmological constant, $\delta_{c c}=0$. The transition from a "dust" phase to a "cosmological constant" phase is smooth and the moment it happens is essentially dictated by the value of the constante $A$ in (2). This behaviour inverts what happens in the traditional case of density perturbations in newtonian gravity with positive pressure 8]. In this case, taking the equation of state $p \propto \rho^{\gamma}, \gamma>4 / 3$, the density contrast initially oscillates, and after it grows with $\delta_{+} \propto t^{2 / 3}$.

These features have some interesting consequences. In fact, let us suppose that at the moment of decoupling of radiation and matter, $t_{i} \sim 10^{11} s$, the amplitude of density perturbations is fixed by the amplitude of the anisotropy of cosmic microwave background radiation, i.e., $\delta_{i} \sim 10^{-5}$. Later, deep in the material phase, the newtonian approximation is reasonable. Supposing the Universe from that moment on is dominated by a Chaplygin gas fluid (in more sophisticated words, a gas of p-branes), than density perturbations grow initially, decreasing later. Observations indicate that the large structure are such today that $\delta_{f}<1$, where the subscript indicates an instant near our now. Supposing the first asymptotic regime for the density contrast found before is valid, than

$$
\frac{\delta_{f}}{\delta_{i}}=\left(\frac{t_{f}}{t_{i}}\right)^{2 / 3}
$$

The condition $\delta_{f}<1$, leads to $t_{f} \sim 10^{17} \mathrm{~s}$, that is $t_{f} \sim t_{0}$, where $t_{0}$ is the age of the Universe. Hence the approximation that the pressure is negligible must be valid 
until very recently in order to explain observations. This fact limits the value of $A$ as $A<\rho_{0}$, where $\rho_{0}$ is the density of the Universe today. Only very recently pressure should become important, and the perturbations have stopped growing. This fact may have some consequences for the anisotropy of cosmic microwave background, at least for large multipoles values, that is, small angles.

In this note, we have outlined the behaviour of density of perturbations in a specific type of fluid with negative pressure, the Chaplygin gas, which can be viewed also as a gas of p-branes. This gas may interpolate a regime of a dust dominated Universe to a vacuum energy dominated Universe, given an explanation for the possible accelerated phase today. We performed a newtonian analysis since the Chaplygin gas obeys the equations of newtonian hydrodynamic. The main conclusion is that it admits an initial phase of growing perturbations, with the the same rate as in dust case of the cosmological standard model, from which it follows decreasing oscillations, which asymptotically go to zero. Hence, even if the newtonian analysis is limited for the study of the Universe in large scales, it this case the newtonian solution interpolates conveniently an initial dust phase $\left(\delta_{d} \propto t^{2 / 3}\right)$ with a cosmological constant phase $\left(\delta_{c c}=0\right)$. Even if the newtonian background model is very different from the relativistic one, since it gives a dust-like solution for any value of time, the perturbative newtonian analysis is consistent with the different phases predicted by expression (1), which comes from relativistic considerations. Moreover, we notice that the Chaplygin gas, in spite of presenting negative pressure, is stable at small scale, in opposition to what happens in general with perfect fluids with negative pressure. This is due to the fact that the sound velocity in the Chaplygin gas is positive.

Acknowledgements: We thank CNPq (Brazil) for partial financial support.

\section{References}

[1] E Battaner and E. Florido, The rotation curve of spiral galaxies and its cosmological implications, astro-ph/0010475;

[2] P.H. Frampton, Quintessence and cosmic microwave background, astro-ph/0102344;

[3] A.G. Riess et al, Observational evidence from supernovae for an accelerating Universe and a cosmological constant, astro-ph/9805201;

[4] S. Permultter et al, Astrophys. J. 517, 565(1998);

[5] A. Kamenshchik, U. Moschella and V. Pasquier, An alternative to quintessence, gr-qc/0103004;

[6] M. Bordemann and J. Hoppe, Phys. Lett. B317, 315(1993);

[7] N. Ogawa, Phys. Rev. D62, 085023(2000);

[8] S. Weinberg, Gravitation and cosmology, Wiley, New York (1972); 
[9] J.C. Fabris and J. Martin, Phys. Rev. D55, 5205(1997);

[10] J.C. Fabris, S.V.B. Gonçalves and N.A. Tomimura, Class. Quant. Grav. 17, 2983(2000); 